\title{
Einstein Dilemma and Two-State Vector Formalism
}

\author{
Kunihisa Morita \\ Faculty of Arts and Science, Kyushu University, Fukuoka, Japan \\ Email: morita@artsci.kyushu-u.ac.jp
}

Received 13 March 2015; accepted 20 May 2015; published 28 May 2015

Copyright (C) 2015 by author and Scientific Research Publishing Inc.

This work is licensed under the Creative Commons Attribution International License (CC BY). http://creativecommons.org/licenses/by/4.0/

(c) (i) Open Access

\section{Abstract}

In the famous EPR paper published in 1935, Einstein, Podolsky, and Rosen suggested a thought experiment, which later became known as the "EPR experiment". Using the EPR experiment, they posited that quantum mechanics was incomplete. Einstein, however, was dissatisfied with the EPR paper and published a second work on the EPR experiment, in which he discussed the dilemma of choosing whether quantum mechanics was incomplete or nonlocal. Currently, most physicists choose the nonlocality of quantum mechanics over Einstein's choice of the incompleteness of quantum mechanics. However, with an appropriate alternate hypothesis, both of these choices can be rejected. Herein, I demonstrate an approach to overcome the Einstein Dilemma by proposing a new interpretation invoked by a new formalism of quantum mechanics known as two-state vector formalism.

\section{Keywords}

Two-State Vector Formalism, Einstein Dilemma, Completeness, Locality

\section{Introduction}

In 1935, Einstein, Podolsky, and Rosen published their famous EPR paper, which posited that the quantum mechanical wave function did not provide a complete description of physical reality ([1], p. 78). However, the EPR paper was factually written by Podolsky, who had submitted the paper without Einstein's validation. Einstein complained about the EPR paper to Schrödinger, stating, "the essential thing was smothered by the formalism” ([2], p. 35). Consequently, Einstein discussed the EPR experiment again and presented the following dilemma [3]:

1) Quantum mechanics is incomplete, or

2) Quantum mechanics is nonlocal. 
This dilemma is at the heart of the EPR experiment. In what follows, we call this dilemma the "Einstein Dilemma” after Redhead ([4], p. 76). Einstein preferred to believe in the incompleteness of quantum mechanics because, in his words,

The total rejection of this principle [principle of action through a medium] would make impossible the idea of the existence of a (quasi-) closed system, and thereby also make impossible the establishment of empirically verifiable laws in our well-known sense ([5], p. 322) (Author’s translation).

Bohr published a paper with the same title as the EPR paper and rebutted the EPR argument [6]. Bohr admitted that "there is, in a case like that, just considered no question of a mechanical disturbance of the system under investigation during the last critical stage of the measuring procedure," but insisted that "even at this stage, there is essentially the question of an influence on the very conditions which define the possible types of predictions regarding the future behavior of the system".

There are many views on Bohr's statement [7]-[11]. However, I believe that regardless of the view taken into consideration, Bohr's argument still could not have circumvented the Einstein Dilemma. Before we discuss Bohr's view, we have to clarify the meaning of the word "locality". Redhead suggests five types of locality that we use in the field of quantum mechanics ([4], p. 77, 82); however, only three of these are related to our discussion:

LOC $_{1}$ : An unsharp value for an observable cannot be changed into a sharp value by measurements performed "at a distance."

$\mathrm{LOC}_{2}$ : A previously undefined value for an observable cannot be defined by measurements performed "at a distance."

$\mathrm{LOC}_{3}$ : A sharp value for an observable cannot be changed into another sharp value by altering the setting of a remotely located apparatus.

Redhead speculates that the locality in the Einstein Dilemma can be described with LOC $_{1}$ and that the locality in Bohr's “complementarity” can be described with LOC $_{2}$. Therefore, Bohr's interpretation could circumvent the Einstein Dilemma because $\mathrm{LOC}_{2}$ does not change real situation. Redhead discussed that the locality $\mathrm{LOC}_{3}$ appears in Bell's theorem.

In this work, I show that the existing interpretations of quantum mechanics, including that of Bohr, must be subject to the Einstein Dilemma (Section 2); further, I provide a new interpretation that makes it possible to circumvent the dilemma (Sections 3 and 4).

\section{Einstein Dilemma and Complementarity}

First, let us examine Redhead's view that Bohr's locality is equivalent to LOC $_{2}$. If this is true, we consider whether Bohr's conclusion can overcome the dilemma.

Let us consider Bohm's version of the EPR experiment ([12], pp. 611-615). Two electrons (I and II), which initially interact with each other at $t_{0}$ and whose total spin value is zero, spatially move apart. When the $x$-spin of electron I is measured at $t\left(t_{0}<t\right)$, we can predict the value of the $x$-spin of electron II with certainty.

If $\mathrm{LOC}_{2}$ is violated, the $x$-spin of electron II is defined by the measurement of the $x$-spin of electron I. Redhead argues that the violation of $\mathrm{LOC}_{2}$ does not mean the violation of $\mathrm{LOC}_{1}$, but is this correct? Defining the spin value of electron I does not guarantee that the $x$-spin of electron II has a sharp value. However, we can predict the $x$-spin of electron II with certainty after $t$, and thus, the $x$-spin has a sharp value. This result suggests that if we accept the violation of $\mathrm{LOC}_{2}$, we also have to accept the violation of $\mathrm{LOC}_{1}$. Therefore, we can conclude that Redhead's version of Bohr's interpretation is subject to the Einstein Dilemma.

We now examine other views of Bohr's interpretation. Beller and Fine discuss that Bohr adopted positivism as his philosophy of science after the EPR paper was published [7] [8]. If Beller and Fine are considered to be correct, this indicates that quantum mechanics violates LOC $_{1}$, and thus, Bohr's interpretation is still subject to the dilemma.

Those familiar with this controversy might consider that Bohr's interpretation could circumvent the dilemma if the view of Howard, Halvorson-Clifton, and Ozawa-Kitajima (HHCOK) of Bohr's interpretation is correct [9]-[11]. HHCOK speculate that the word "classical” as used by Bohr refers to the mixture state and that the experimental setup determines how a pure state changes into the mixture state. We can say that (according to HHCOK) Bohr introduces “nonseparability” instead of “nonlocality” (see also [13]). 
According to the HHCOK version of Bohr's view, before the measurement of electron I, the states of the electrons are the mixture states of the $x$-spin. It should be noted, however, that the mixture state of the $x$-spin is not the eigenstate of the $x$-spin, and thus, we cannot predict which value the $x$-spin would assume using only quantum mechanics. This assumption suggests that we have to choose one of the following options:

1) The $x$-spin has a sharp value before the measurement. It follows that quantum mechanics is incomplete because it cannot predict which value the $x$-spin would have before measurement (since the mixture state is not the eigenstate).

2) The $x$-spin has an unsharp value before the measurement. It follows that quantum mechanics violates LOC $_{1}$ because the spin of electron II can have a sharp value only after the measurement of the spin of electron I.

Therefore, the HHCOK version of Bohr's interpretation of quantum mechanics will also face the Einstein Dilemma. Accordingly, it does not matter which view of Bohr's interpretation we consider, we cannot reject either part of the dilemma. Next, we examine the existing interpretations of quantum mechanics other than Bohr's interpretation.

HHCOK speculate that Bohr's interpretation does not accept the projection postulate. If we consider an interpretation that assumes the projection postulate, it follows that quantum mechanics is nonlocal (violation of $\mathrm{LOC}_{1}$ ). The de Broglie-Bohm interpretation concludes that quantum mechanics is incomplete (and nonlocalviolation of $\mathrm{LOC}_{3}$ ).

The many-worlds interpretation (MWI) postulates that quantum mechanics is nonlocal. Some readers might believe the MWI avoids the issue of nonlocality. Nevertheless, because the worlds split for a very short period of time, we cannot avoid nonlocality [14]. Although this interpretation does not necessarily mean the violation of localities $\mathrm{LOC}_{1}-\mathrm{LOC}_{3}$, it does mean that the real situation is widely changed for a very short period of time. Einstein's meaning of "nonlocal" is the action that telepathically changes the real situation ([3], p. 322) ([5], p. 85), and thus, the MWI is also subject to the Einstein Dilemma.

Next, we consider whether it is impossible to avoid the Einstein Dilemma. In the remainder of this paper, I suggest an interpretation that circumvents the dilemma. This interpretation is based on one of the formalisms of quantum mechanics, known as "two-state vector formalism (TSVF)," as proposed by Aharonov, Bergmann and Leibowitz (ABL) [15]. I briefly summarize TSVF in Section 3, and I show how the new interpretation makes it possible to circumvent the Einstein Dilemma in Section 4.

\section{Brief Review of Two-State Vector Formalism}

In this section, I briefly summarize TSVF. In conventional quantum mechanics (CQM), only the past state determines the probability that a physical quantity $Q$ has a certain value $q$ (the Born rule). In contrast, in TSVF, both the past and the future states determine the probability that $Q$ has a certain value $q$. The ABL rule is used for determining the probability from the past and the future states [15].

Assume that $Q$ has eigenstates $\left|q_{1}\right\rangle,\left|q_{2}\right\rangle, \cdots,\left|q_{n}\right\rangle$, which, respectively, have eigenvalues $q_{1}, q_{2}, \cdots, q_{n}$ (assume that there is no degeneracy). The probability, $P\left(q_{i}, t\right)$, that $Q$ has a value $q_{i}$ at $t$ is calculated as follows using the ABL rule:

$$
P\left(q_{i}, t\right)=\frac{\left|\left\langle\Psi_{\text {fin }}(t) \mid q_{i}\right\rangle\left\langle q_{i} \mid \Psi_{\text {ini }}(t)\right\rangle\right|^{2}}{\sum_{k}\left|\left\langle\Psi_{\text {fin }}(t) \mid q_{k}\right\rangle\left\langle q_{k} \mid \Psi_{\text {ini }}(t)\right\rangle\right|^{2}}
$$

Here,

$$
\begin{aligned}
& \left|\Psi_{\text {fin }}(t)\right\rangle=\exp \left[i \hat{H}\left(t_{1}-t\right)\right]\left|\Psi_{\text {fin }}\left(t_{1}\right)\right\rangle, \\
& \left|\Psi_{\text {ini }}(t)\right\rangle=\exp \left[-i \hat{H}\left(t_{0}-t\right)\right]\left|\Psi_{\text {ini }}\left(t_{0}\right)\right\rangle,
\end{aligned}
$$

where $\left|\Psi_{\text {ini }}\left(t_{0}\right)\right\rangle$ denotes the initial state of the system measured at $t_{0}$, and $\left|\Psi_{\text {fin }}\left(t_{1}\right)\right\rangle$ denotes the final state of the system measured at $t_{1}$, where $t_{0}<t<t_{1}$.

At times, the probabilities calculated using the $\mathrm{ABL}$ rule differ from those calculated using the Born rule. It appears as if TSVF and CQM are different theories, although they are not. The ABL rule agrees with the Born rule when $\left|\Psi_{\text {fin }}(t)\right\rangle$ in Equation (1) changes to $\left|\Psi_{\text {ini }}(t)\right\rangle$; this implies that TSVF agrees with CQM when we consider only the past state. 
There seems to be no method for verifying the prediction made using TSVF because we cannot know the intermediate state at $t$ without destroying the state. Aharonov, Albert and Vaidman solved this problem by proposing a new measurement concept called 'weak measurement'; measurements made according to this concept do not destroy the intermediate quantum state [16]. In the remainder of this paper, we refer to the conventional measurement as 'strong measurement'.

According to Aharonov, Albert and Vaidman [17], the mean value obtained by weak measurement is the "weak value". The weak value of the physical quantity $Q$ at $t, Q_{w}(t)$, is

$$
Q_{w}(t)=\frac{\left\langle\Psi_{\text {fin }}(t)|Q| \Psi_{\text {ini }}\left(t_{0}\right)\right\rangle}{\left\langle\Psi_{\text {fin }}(t) \mid \Psi_{\text {ini }}\left(t_{0}\right)\right\rangle}
$$

Recently, physicists performed a weak measurement and confirmed that the theoretically predicted and measured weak values showed good agreement [18].

\section{Einstein Dilemma and Two-State Vector Formalism}

In general, when the final state is an eigenstate, $\left|q_{i}\right\rangle$, of $Q$, the intermediate state is also $\left|q_{i}\right\rangle$ with certainty because

$$
P\left(q_{i}, t\right)=\frac{\left|\left\langle q_{i} \mid q_{i}\right\rangle\left\langle q_{i} \mid \Psi_{\text {ini }}(t)\right\rangle\right|^{2}}{\sum_{k}\left|\left\langle q_{i} \mid q_{k}\right\rangle\left\langle q_{k} \mid \Psi_{\text {ini }}(t)\right\rangle\right|^{2}}=1
$$

Thus, the physical quantity has a sharp value even before the measurement. In this paper, I do not consider the case where the final state is not an eigenstate. Consider Bohm's version of the EPR experiment. When the interaction between two electrons ends at $t_{0}$, the state of the whole system is

$$
\left|\Psi_{\text {ini }}\right\rangle=\left(|+x\rangle_{\mathrm{I}}|-x\rangle_{\mathrm{II}}+|-x\rangle_{\mathrm{I}}|+x\rangle_{\mathrm{II}}\right) / \sqrt{2}
$$

where $|+x\rangle$ and $|-x\rangle$ represent the states in which the measured values of the $x$-spin are $+1 / 2$ and $-1 / 2$, respectively (unit is $\hbar$ ). When the $x$-spin of electron I is measured at $t_{1}$ and the value is $+1 / 2$, the state of the system becomes

$$
\left|\Psi_{\text {fin }}\right\rangle=|+x\rangle_{\mathrm{I}}|-x\rangle_{\mathrm{II}}
$$

From the ABL rule (1), the probability that the state of the system at a given time $t \quad\left(t_{0}<t<t_{1}\right)$ is $|+x\rangle_{\mathrm{I}}|-x\rangle_{\mathrm{II}}$ is 1 . Therefore, the values of the $x$-spin of electrons I and II are $+1 / 2$ and $-1 / 2$, respectively, subsequent to the end of the interaction.

It follows from this result that we do not need to assume any type of nonlocality. Furthermore, quantum mechanics is not modified. Accordingly, we can simultaneously insist that quantum mechanics is complete and local. Here, we can avoid confronting the Einstein Dilemma.

Readers might suspect that using TSVF means that quantum mechanics is not complete because the final state can be considered a "hidden variable." Actually, it depends on the definition of the hidden variable. For example, the de Broglie-Bohm interpretation needs hidden variables of both position and momentum because we can clearly interpret that quantum mechanics does not need both position and momentum.

However, it is not always clear whether the final state is a hidden variable, unlike the case with the de Broglie-Bohm interpretation. TSVF formalism does not modify quantum mechanics because TSVF considers the description of the state vector as complete, but we need two state vectors for completeness. TSVF does not require adding any other variables other than the state vector. Therefore, I insist that quantum mechanics is still complete if my interpretation using TSVF is correct.

Nevertheless, this interpretation still appears problematic in the following situation. Let us assume that we measure the $z$-spin of an electron at $t_{0}$ and obtain a value $+1 / 2$; thus, the system state is $|+z\rangle$. Thereafter, we measure the $x$-spin of the electron at $t_{1}$ and obtain a value $+1 / 2$; thus, the system state is $|+x\rangle$.

According to the ABL rule, both the probability that the system state at $t \quad\left(t_{0}<t<t_{1}\right)$ is $|+z\rangle$ is 1 and the probability that the system state at $t$ is $|+x\rangle$ is 1 . However, a quantum system cannot have eigenstates of both $x$-spin and $z$-spin simultaneously. Let us assume that the state of a system $(|A\rangle)$ is an eigenstate of 
non-commutative observables $Q_{1}$ and $Q_{2}$ having eigenvalues $q_{1}$ and $q_{2}$, respectively. Consequently,

$$
\left(Q_{1} Q_{2}-Q_{2} Q_{1}\right)|A\rangle=\left(q_{1} q_{2}-q_{2} q_{1}\right)|A\rangle=0
$$

This contradicts the assumption that $Q_{1}$ and $Q_{2}$ are non-commutative. Therefore, Aharonov, Popescu, and Tollaksen state the following:

If at $t$ we measure the spin in the $z$-direction, we must find it up, because that's how the particle was prepared at $t_{0}$. On the other hand, if at $t$ we measure the spin along $x$, we must also find it up, because otherwise the measurement at $t_{1}$ wouldn't find it up ([19], p. 27).

Nonetheless, there is still concern that our interpretation contradicts the uncertainty relation (Kennard-Robertson inequality). Let us assume that an ensemble of electrons whose $z$-spin at $t_{0}$ is $+1 / 2$ and $x$-spin at $t_{1}$ is $+1 / 2$ has been prepared, and let us divide the ensemble into two sub-ensembles: $\Sigma_{1}$ and $\Sigma_{2}$. When we perform weak measurement of the $x$-spin in $\Sigma_{1}$ and the $z$-spin in $\Sigma_{2}$ at $t$, we would obtain the result that the $x$-spin is $+1 / 2$ and $z$-spin is $+1 / 2$ with certainty. This result indicates that both the standard deviations of $x$-spin in $\Sigma_{1}$ and $z$-spin in $\Sigma_{2}$ are 0 . This result appears to contradict the uncertainty relation.

However, it is to be noted that the uncertainty relation considers only the past state. In our interpretation, we consider the future state as well, thus it is no problem to contradict with the uncertainty relation.

Here, we define new standard deviation of $z$-spin for our interpretation as

$$
\begin{aligned}
& \left(\Delta_{w} S_{z}\right)^{2}=\frac{\left\langle+z\left|S_{z}^{2}\right| \Psi_{z}\right\rangle}{\left\langle+z \mid \Psi_{z}\right\rangle}-\left|\frac{\left\langle+z\left|S_{z}\right| \Psi_{z}\right\rangle}{\left\langle+z \mid \Psi_{z}\right\rangle}\right|^{2}, \\
& \left|\Psi_{z}\right\rangle=(|+z\rangle+|-z\rangle) / \sqrt{2} .
\end{aligned}
$$

where $S_{z}$ denotes an operator of $z$-spin. Consequently, we can easily obtain

$$
\left(\Delta_{w} S_{z}\right)^{2}=0 \text {. }
$$

Likewise, we can obtain $\left(\Delta_{w} S_{x}\right)^{2}=0$. Therefore, when we interpret $\Delta_{w} S_{z}$ as the standard deviation of $z$ spin in TSVF, it is not starange that both of the standard deviations of $x$-spin in $\Sigma_{1}$ and z-spin in $\Sigma_{2}$ are 0 .

In general, when we define

$$
\begin{aligned}
& \left(\Delta_{w} Q\right)^{2}=\left(Q^{2}\right)_{w}-\left(Q_{w}\right)^{2}, \\
& \left|\Psi_{\text {ini }}\right\rangle=a_{1}\left|\phi_{1}\right\rangle+a_{2}\left|\phi_{2}\right\rangle+\cdots+a_{k}\left|\phi_{k}\right\rangle+\cdots+a_{n}\left|\phi_{n}\right\rangle,
\end{aligned}
$$

where $Q$ is an observable quantity, $\left|\phi_{i}\right\rangle$ represents an eigenstate of $Q$, and $\left\langle\phi_{i} \mid \phi_{j}\right\rangle=\delta_{i j}$, we can easily obtain

$$
\left(\Delta_{w} Q\right)^{2}=0
$$

when the final state is an eigenstate of $Q$.

Nevertheless, there remains the concern that the abovementioned interpretation may contradict no-go theorem such as the Kochen-Specker theorem [20]. However, we can easily infer from Mermin's version of the KochenSpecker theorem that there might not be any contradiction when only $x$-spin and $z$-spin are determined because the Kochen-Specker theorem holds only when more than two axes of spin are determined [21].

In addition, Tollaksen discusses that the weak value of spin changes according the context of the experiment [21] [22]. For example, he shows that there is a case where $\left(S_{x}^{1} S_{y}^{2} S_{x}^{2} S_{y}^{1}\right)_{w}=-1$, even though $\left(S_{x}^{1} S_{y}^{2}\right)_{w}=+1$ and $\left(S_{x}^{2} S_{y}^{1}\right)_{w}=+1$, where $\left(S_{i j}\right)_{w}$ represents the weak value of $j$-spin $(j=x, y)$ of particle $i \quad(=1,2)$.

\section{Conclusions}

Upon his examination of quantum mechanics in the EPR paper, Einstein was presented with the dilemma that

1) Quantum mechanics is incomplete, or

2) Quantum mechanics is nonlocal.

Most physicists choose the nonlocality of quantum mechanics over Einstein's choice of incompleteness. 
However, if possible, it is better to reject both choices.

According to TSVF, physical quantities can have sharp values before measurement. Therefore, we do not need to introduce the concept of nonlocality. Furthermore, TSVF does not assume any hidden variables; thereby ensuring that quantum mechanics is complete. In conclusion, we circumvented the Einstein Dilemma by considering both the past state and the future state of a quantum mechanical system.

\section{Acknowledgements}

This work has been supported by Japan Society for the Promotion of Science (Grant No. 26370021).

\section{References}

[1] Einstein, A., Podolsky, B. and Rosen, N. (1935) Can Quantum-Mechanical Description of Physical Reality Be Considered Complete? Physical Review, 47, 777-780. http://dx.doi.org/10.1103/PhysRev.47.777

[2] Fine, A. (1986) The Shaky Game. University of Chicago Press, Chicago.

[3] Einstein, A. (1948) Quanten-Mechanik und Wirklichkeit. Dialectica, 2, 320-324. http://dx.doi.org/10.1111/j.1746-8361.1948.tb00704.x

[4] Redhead, M. (1987) Incompleteness, Nonlocality and Realism. Clarendon Press, Oxford.

[5] Einstein, A. (1949) Autobiographical Notes. In: Schilp, P.A., Ed., Albert Einstein: Philosopher-Scientist, Open Court, New York, 1-94.

[6] Bohr, N. (1935) Can Quantum-Mechanical Description of Physical Reality Be Considered Complete? Physical Review, 48, 696-702. http://dx.doi.org/10.1103/PhysRev.48.696

[7] Beller, M. and Fine, A. (1993) Bohr’s Response to EPR. In: Faye, J. and Folse, H.J., Eds., Niels Bohr and Contemporary Philosophy, Kluwer Academic Publishers, New York, 1-31.

[8] Fine, A. (2007) Bohr's Response to EPR: Criticism and Defense. Iyyun: The Jerusalem Philosophical Quarterly, 56, 1-26.

[9] Howard, D. (1994) What Makes a Classical Concept Classical? In: Faye, J. and Folse, H.., Eds., Niels Bohr and Contemporary Philosophy, Kluwer Academic Publishers, New York, 201-229. http://dx.doi.org/10.1007/978-94-015-8106-6_9

[10] Halvorson, H. and Clifton, R. (2002) Reconsidering Bohr’s Reply to EPR. In: Butterfield, J. and Placek, T., Eds., Modality, Probability and Bell's Theorems, Kluwer Academic Publishers, Dordrecht, 3-18.

[11] Ozawa, M. and Kitajima, Y. (2012) Reconstructing Bohr’s Reply to EPR in Algebraic Quantum Theory. Foundations of Physics, 42, 475-487. http://dx.doi.org/10.1007/s10701-011-9615-7

[12] Bohm, D.J. (1951) Quantum Theory. Dover Publications, New York.

[13] Howard, D. (1989) Holism, Separability, and the Metaphysical Implication of the Bell Experiment. In: Cushing, J.T. and McMullin, E., Eds., Philosophical Consequences of Quantum Theory: Reflection on Bell's Theorem, University of Notre Dame Press, Notre Dame, 224-253.

[14] Vaidman, L. (2002) Many-Worlds Interpretation of Quantum Mechanics. In: Zalta, E.N., Ed., Stanford Encyclopedia of Philosophy. http://plato.stanford.edu

[15] Aharonov, Y., Bergmann, P.G. and Lebowitz, J.L. (1964) Time Symmetry in the Quantum Process of Measurement. Physical Review B, 134, 1410-1416. http://dx.doi.org/10.1103/PhysRev.134.B1410

[16] Aharonov, Y., Albert, D.Z. and Vaidman, L. (1988) How the Result of a Measurement of a Component of the Spin-1/2 Particle Turn Out to Be 100. Physical Review Letters, 60, 1351-1354. http://dx.doi.org/10.1103/PhysRevLett.60.1351

[17] Aharonov, Y. and Vaidman, L. (1990) Properties of a Quantum System during the Time Interval between Two Measurements. Physical Review A, 41, 11-20. http://dx.doi.org/10.1103/PhysRevA.41.11

[18] Yokota, K., Yamamoto, T., Koashi, M. and Imoto, N. (2009) Direct Observation of Hardy’s Paradox by Joint Weak Measurement with an Entangled Photon Pair. New Journal of Physics, 11, Article ID: 033011. http://dx.doi.org/10.1088/1367-2630/11/3/033011

[19] Aharonov, Y., Popescu, S. and Tollaksen, J. (2010) A Time-Symmetric Formulation of Quantum Mechanics. Physics Today, 63, 27-32. http://dx.doi.org/10.1063/1.3518209

[20] Kochen, S.B. and Specker, E.P. (1967) The Problem of Hidden Variables in Quantum Mechanics. Journal of Mathematics and Mechanics, 17, 59-87. http://dx.doi.org/10.1512/iumj.1968.17.17004

[21] Mermin, N.D. (1993) Hidden Variables and the Two Theorems of John Bell. Reviews of Modern Physics, 65, 803-815. http://dx.doi.org/10.1103/RevModPhys.65.803

[22] Tollaksen, J. (2007) Pre- and Post-Selection, Weak Values and Contextuality. Journal of Physics A: Mathematical and Theoretical, 40, 9033-9066. http://dx.doi.org/10.1088/1751-8113/40/30/025 